\title{
Low Altitude UAV Air-to-Ground Channel Measurement and Modeling in Semiurban Environments
}

\author{
Zhihong Qiu, ${ }^{1}$ Xi Chu, ${ }^{1}$ Cesar Calvo-Ramirez, ${ }^{2}$ César Briso, ${ }^{2}$ and Xuefeng Yin ${ }^{1}$ \\ ${ }^{1}$ College of Electronics and Information Engineering, Tongji University, Shanghai 201804, China \\ ${ }^{2}$ School of Systems and Telecommunications Engineering, Technical University of Madrid, 28031 Madrid, Spain \\ Correspondence should be addressed to Zhihong Qiu; qiu_zhihong@tongji.edu.cn
}

Received 26 June 2017; Revised 18 September 2017; Accepted 8 October 2017; Published 13 November 2017

Academic Editor: Daniele Pinchera

Copyright (c) 2017 Zhihong Qiu et al. This is an open access article distributed under the Creative Commons Attribution License, which permits unrestricted use, distribution, and reproduction in any medium, provided the original work is properly cited.

\begin{abstract}
Small- and medium-sized unmanned aerial vehicles (UAVs) can fly for a short distance $(<2 \mathrm{~km})$ from a control station in a nonsegregated air space (altitudes $<100 \mathrm{~m}$ ). It is of great interest to model the propagation channel under such condition, where there is an important influence from the environment. This paper presents multiple measurements carried out in low altitudes with a medium-sized UAV flying over a semiurban environment. Path loss exponent is given based on the measurements done at different altitudes and a height-dependent Rician $K$ factor model is proposed. The results clearly reveal the existence of two propagation zones with very distinct channel characteristics. The breakpoint indicates the height where the condition of the channel changes rapidly. At low altitudes, the obstacles generate a large amount of multipath and the propagation is greatly affected, while at higher altitudes the influence mitigates. Our results are useful for the modeling of low altitude air-to-ground (AG) propagation channels and the performance analysis of UAV-enabling AG communication systems, such as the channel capacity and the throughput.
\end{abstract}

\section{Introduction}

The usage of unmanned aircraft systems (UASs) has been increasing significantly in recent years, and a strong growth of commercial applications is expected in the coming years [1]. The majority of UASs are expected to be small and microUASs, which are also called "drones." Most commercial drones are usually multiple rotor copters with a structure different from that of airplanes, which allows them to easily take off without a long runway. Drones are slow but more flexible and have high mobility dynamics with the ability to fly in all directions in 3D space. In most countries, these UASs are facing more and more regulations. For instance, in the USA and Europe, civil drones can only fly without exceeding the regulated altitude $(100 \mathrm{~m})$ and distance $(<1 \mathrm{~km})$ in nonsegregated air spaces and must be under direct visual line of sight of the operator $[2,3]$.

For civil applications, UAVs are promising in numerous areas such as agriculture, industry, scientific surveys, emergency communications, safety and rescue, package transportation, and video filming. These applications raise high requirements on high quality communication links for control and high capacity links for the transmission of payload information. Also, UASs must be operative in complex environments such as urban areas and industrial scenarios. For those communication scenarios, part of the L-band $(1-1.2 \mathrm{GHz})$ is currently being proposed for the control link and the C-band $(4-6 \mathrm{GHz})$ for the payload [4]. Thus, it is necessary to carefully model the air-to-ground $(\mathrm{AG})$ channel at low altitudes in these frequency bands.

The AG channel has been extensively modeled and characterized in multiple experiments. Generally, the AG channel has been treated as either a free-space channel or a two-ray channel [5] that adds a reflection from the earth's surface to the direct or line-of-sight (LoS) component. Conventional AG channel measurements and modeling were made with large aircraft at high altitudes using fixed wing airplanes [6], balloons [7], or airships [8]. However, the heights and flight dynamics of those measurements are different and inadequate to describe the influence of the ground environment on the propagation channel at low heights. Only a few measurements conducted at low altitudes can be found in 
the literature [9], yet the altitudes are still higher than 200 $\mathrm{m}$. Therefore, it is necessary to investigate and model the propagation channel of small UAVs at heights from 0 to $100 \mathrm{~m}$. In this range of heights, the propagation is seriously affected by the environment and the heights of the adjoining obstacles. There is an increase of the average path losses due to deep fast fading. Some reports concerned with this phenomenon at low altitudes have been given $[8,10]$ and simulations made by ray tracing methods [11] can explain the effects of the reflection, diffraction, and scattering of the environment on low altitude AG channels.

In [12], the author conducted measurements at different distances and altitudes with a small UAV using 802.11 networks, where an interesting phenomenon of the impact of height on the propagation channel was observed. In [13], a height-dependent model based on ray tracing methods has been proposed. However, more literature focuses on the elevation angle of the link distance of UAVs. In [14], the aircraft was flying circularly around the base station to investigate the impact of the angle. In [11, 15], ray tracing methods were applied to investigate the propagation channel in urban environments, and in [16] wideband measurements were conducted with a UAV being fixed at a certain position, which depicts the influence of the ground. In [17], narrowband and wideband channel measurements were introduced using LTE signals. In general, there is still limited literature on modeling at ultralow altitude for small UAVs.

In our case, we have investigated the variations of the propagation channel within the flying range of small- and medium-sized UAVs. Measurements of different flight routes in a semiurban complex environment have been done to obtain data of different positions. The analysis of measurement results shows that small-scale fading has high dependency on the flying height instead of the elevation angle or the distance. The structure of this paper is organized as follows. Section 2 introduces the measurement equipment configuration and details of the measurement campaign. Section 3 analyzes the data and proposes a path loss model and a height-dependent Rician $K$ factor model. Then, Section 4 gives a simulation of the model proposed. Conclusions are given in Section 5.

\section{Measurement}

2.1. Measurement System. The testbed is composed of a light continuous wave transmitter installed at the lower part of a medium-sized UAV. The test transmitter can work in L- and $\mathrm{C}$-bands with a maximum transmitting power of $30 \mathrm{dBm}$. The frequencies used for measurements are $1200 \mathrm{MHz}$ for L-band and $4200 \mathrm{MHz}$ for C-band, respectively. The transmitting antennas used on the UAV are two cloverleaf antennas, working in L- and C-bands, respectively. These antennas have circular polarization with an omnidirectional radiation pattern on the horizontal plane and a $3 \mathrm{~dB}$ beamwidth of $60^{\circ}$ for L-band and $55^{\circ}$ for C-band. The antennas were installed on the bottom of the UAV pointing straight down as shown in Figure 1 to minimize interferences from the UAV structure. In our case, the UAV used has a very good azimuthal stability so that we could have used even linear polarization in
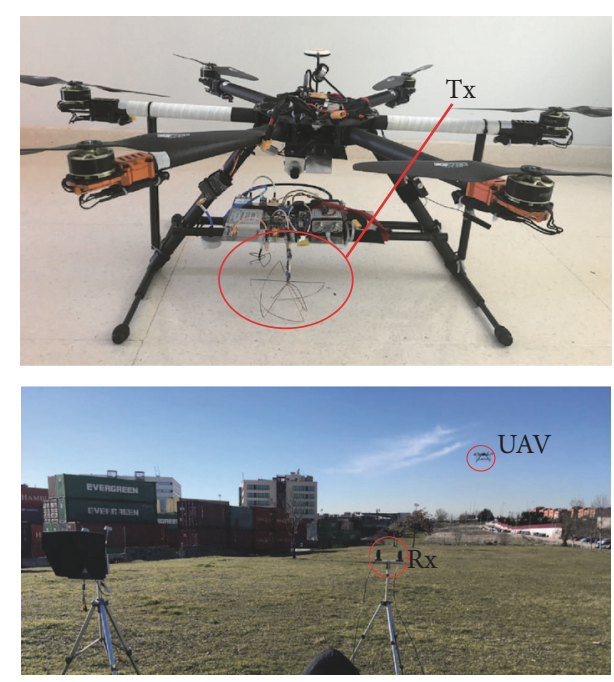

FIGURE 1: UAV and the test environment.

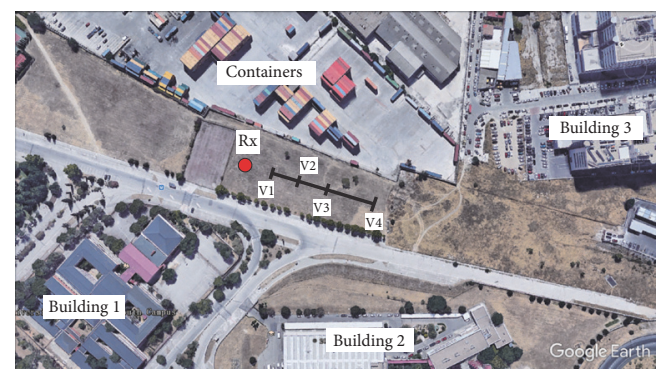

FIGURE 2: Measurement environment from Google Earth.

the UAV with a minimum error. Nevertheless, cloverleaf circularly polarized antennas are widely used for small UAVs and we can minimize the polarization errors using circular polarization in the UAV and linear polarization in the ground station.

The receiving system consists of two antennas of type MGRM-WHF with $20 \mathrm{~cm}$ separation. The antenna is vertically polarized with a gain of $3-5 \mathrm{~dB}$ and its half-power beamwidth in elevation is $50^{\circ}$. The antennas were installed on a $1.5 \mathrm{~m}$ mast. The $\mathrm{Rx}$ was a standard portable spectrum analyzer with customized software to record measurements at a speed of 10 measurements per second. The receiver has two channels and each was connected to one antenna. Both channels received the same signal.

The UAV is a hexacopter of $3 \mathrm{~kg}$ weight, which is able to carry a $2 \mathrm{~kg}$ payload and has 20 minutes of flying endurance. The system was controlled with a $2.4 \mathrm{GHz}$ link and was monitored with a ground station where the telemetry data of height, speed, and position were received. The flights were made with computer-assisted control software to guarantee the accuracy of the trajectories.

2.2. Measurement Campaigns. The measurements were conducted in a suburban open environment as depicted in Figure 2, where there are many buildings, metal containers, and trees. This environment is a multipath-rich environment so that we can easily distinguish the influence of the flying 
TABLE 1: Measurement trajectory details.

\begin{tabular}{lccc}
\hline Trajectory & Direction & Distance & Height \\
\hline V1 & Vertical & $20 \mathrm{~m}$ & $0-25 \mathrm{~m}$ \\
V2 & Vertical & $40 \mathrm{~m}$ & $0-50 \mathrm{~m}$ \\
V3 & Vertical & $60 \mathrm{~m}$ & $0-50 \mathrm{~m}$ \\
V4 & Vertical & $100 \mathrm{~m}$ & $0-100 \mathrm{~m}$ \\
H1 & Horizontal & $20-90 \mathrm{~m}$ & $10 \mathrm{~m}$ \\
H2 & Horizontal & $20-90 \mathrm{~m}$ & $30 \mathrm{~m}$ \\
\hline
\end{tabular}

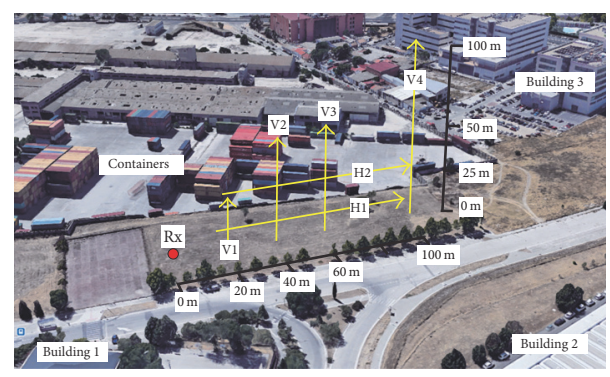

FIGURE 3: Flight trajectories.

height on the propagation. Measurements were made with vertical and horizontal flights. Vertical flight trajectories, as shown with yellow lines in Figure 3, were conducted at an altitude from 0 to $100 \mathrm{~m}$ with the UAV taking off and landing at several fixed distances from $20 \mathrm{~m}$ to $100 \mathrm{~m}$ away from the $\mathrm{Rx}$, and the horizontal position was stabilized. Vertical flights guarantee that the influence of the environment remains constant and can accurately model the influence of the height with negligible influence from the antennas. Several horizontal flights were also made with the UAV starting at a position $20 \mathrm{~m}$ away from $\mathrm{Rx}$, following a straight line forward with constant altitude at $10 \mathrm{~m}$ and $30 \mathrm{~m}$, and landing at a position $90 \mathrm{~m}$ away from the $\mathrm{Rx}$.

The vertical ascending speed was monitored on the ground station as $0.7 \mathrm{~m}$ per second and the horizontal forward speed was around $1.2 \mathrm{~m}$ per second. The same flights were made for L-band and C-band measurements. All flights ensure that the LoS link between the Tx and the Rx is within the range of the antenna radiation patterns. Details of the measurement trajectories are shown in Table 1. To be clear, we use "V1, V2,..." to represent the specific measurement trajectory in the rest of this paper.

2.3. Calibration. In this section, the radiation patterns of antennas were measured in an anechoic chamber of $6 \times 3 \times$ $3 \mathrm{~m}^{3}$, in order to calibrate the antenna's influence on the complete site.

The UAV was installed on a rotating positioner with the transmitting antennas placed on the bottom of it. Both azimuth and elevation planes were measured for the two cloverleaf antennas, one for $1.2 \mathrm{GHz}$ and one for $4.2 \mathrm{GHz}$, respectively. Depicted in Figure 4(a) are the measured radiation patterns. It can be observed that the elevation radiation patterns of these antennas have more than $60^{\circ}$ of $3 \mathrm{~dB}$ beamwidth with a uniform response and have not
TABLE 2: Path loss exponent $n$.

\begin{tabular}{lcccc}
\hline & \multicolumn{2}{c}{ C-band } & \multicolumn{2}{c}{ L-band } \\
\hline Height & $10 \mathrm{~m}$ & $30 \mathrm{~m}$ & $10 \mathrm{~m}$ & $30 \mathrm{~m}$ \\
$n$ & 2.7631 & 2.6433 & 3.3464 & 2.9889 \\
\hline
\end{tabular}

been influenced by the UAV. In Figure 4(b), we present the measurements of the base station antennas, which are commercial antennas. We present only measurements of the elevation planes with the base station antennas installed on the mast we used. As we can see, the radiation patterns can be used with minor error for directions with elevations up to $60^{\circ}$.

Therefore, we can postulate that the measurements are accurate until $60^{\circ}$ elevation angle for the UAV. In fact, the maximum elevation that we consider in the campaign is up to $45^{\circ}$, which is much less than $60^{\circ}$. The results also show that the antenna radiation patterns exhibit smooth gains in the azimuth-elevation ranges considered for acquiring the data, and thus the measured channel properties are mainly with respect to the propagation characteristics with negligible impact from the antennas.

\section{Data Analysis and Results}

3.1. Path Loss Modeling. The horizontal measurements are used to determine the path loss exponent (PLE) $n$ of the conventional path loss model in this environment, in which $\mathrm{PL}(D)$ results as follows:

$$
\mathrm{PL}(D)=\operatorname{PL}\left(D_{0}\right)+10 n \log _{10}\left(\frac{D}{D_{0}}\right)+X_{\sigma}(K),
$$

where $D_{0}$ denotes the reference distance and $D$ represents the link distance between the Tx and the $\operatorname{Rx}$. $D$ equals $\sqrt{d^{2}+h^{2}}$, where $d$ is the horizontal distance between Tx and Rx and $h$ is the height of the UAV. $X_{\sigma}(K)$ is the small-scale fading following Rician distribution with different $K$. The values of $n$ are given in Table 2 .

Hence, the fittings of the path loss model based on horizontal flights at $10 \mathrm{~m}$ (measurement $\mathrm{H1}$ ) and at $30 \mathrm{~m}$ (measurement H2) for L-band/C-band are given in Figure 5. The PLEs $n$ are from 2.64 to 3.35, which are typical values for suburban areas.

It is interesting that the value of $n$ decreases when the UAV height increases, which is also observed in measurements V3 and V4 shown in Figure 6. It is noted that, in [18], the PLE is also found to decrease with the increase of the relative height of the base station in railway viaduct scenarios, and in [19] the PLE $n$ is measured and found to be less than 2 and is close to free space when UAV is at an altitude higher than $500 \mathrm{~m}$ in a suburban environment. Thus, we consider that the path loss has a tendency to decline with the increasing of UAV heights at low altitude, while at a high altitude it approaches free space. This phenomenon could be the consequence of the reduction of multipath during the ascending process of the UAV.

3.2. Analysis of the Vertical Measurements. We have observed that the properties of the channel remain stable when the 


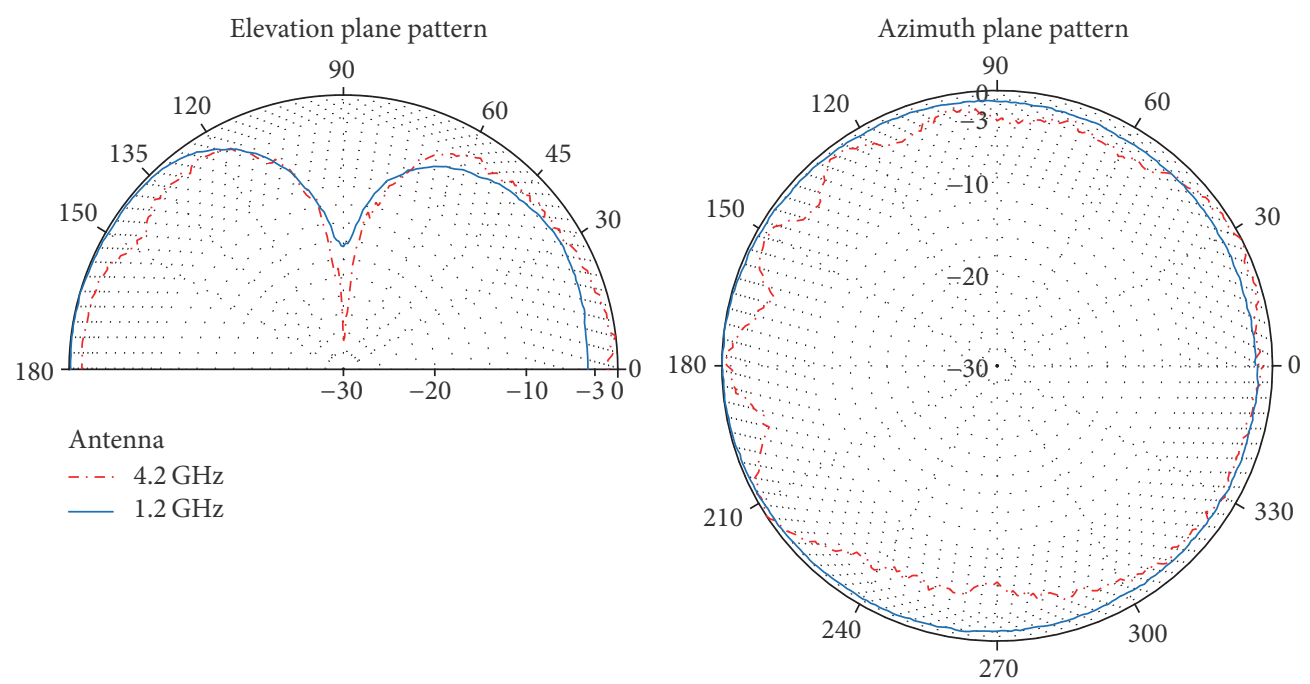

(a) Transmitting antenna

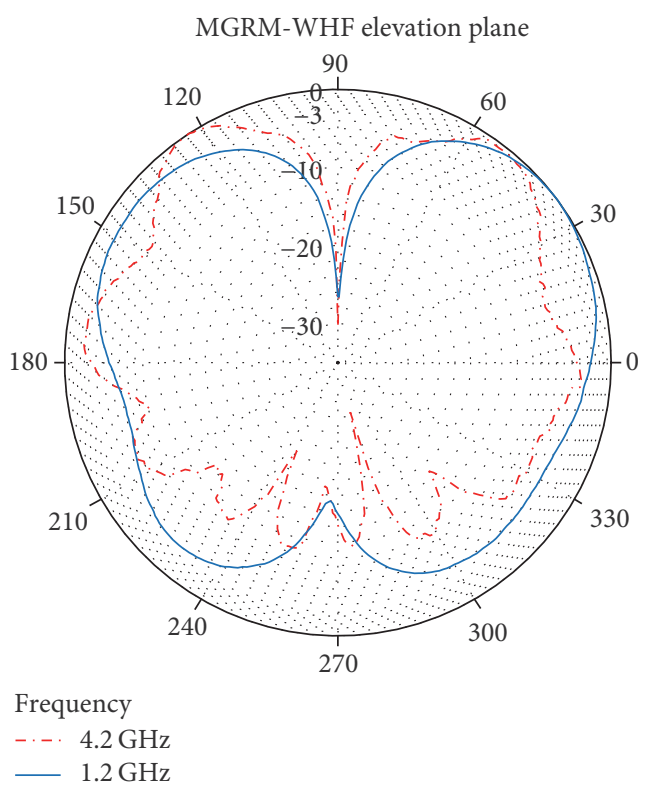

(b) Receiving antenna

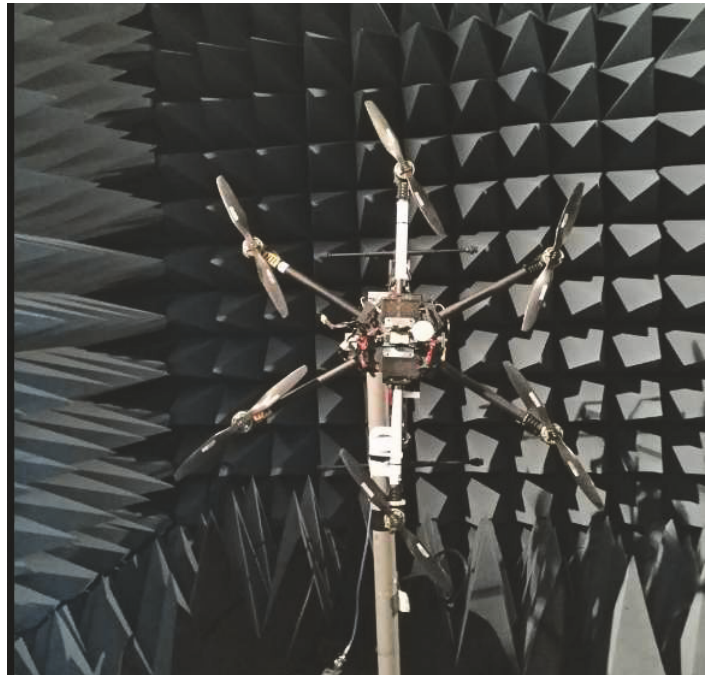

(c) UAV with the transmitting antennas

FIGURE 4: Radiation patterns of the antennas.

UAV flies within short ranges at a constant height. Thus, vertical measurements were made to investigate the propagation channel with respect to height.

Illustrated in Figure 6 are the results of measurements $\mathrm{V} 3$ and V4. It is observed that the vertical measurements conducted at different distances away from $\mathrm{Rx}$ show high similarity. Strong fading is observed in both horizontal and vertical measurements at low altitudes, which shows high dependency on UAV's heights. As observed in Figure 6, there are two obvious propagation zones. There are deep fading and extra losses in zone 1, while in zone 2 the power is more similar to free-space channel. Therefore, we have divided the channel into two zones defined by a "breakpoint" BP, which is around $45 \mathrm{~m}$ in our test environment.
It is noted that the observations about $\mathrm{BP}$ in narrowband measurements in this paper are different from the preliminary work in [17]. This is because the height of the base station antennas applied in the preliminary work was around $50 \mathrm{~cm}$, which was so low that there was an influence of the first Fresnel zone. However, in this work, the antennas were installed on a $1.5 \mathrm{~m}$ high mast, and thus the effect of the first Fresnel zone was minimized. Of course, there were other minor things; that is, the signal-to-noise (SNR) ratio in the preliminary work was not so high, which also could result in such differences.

In this work, the measurement equipment was well calibrated and the influence of antennas has been considered. Also, the measurements have been repeated several times in 

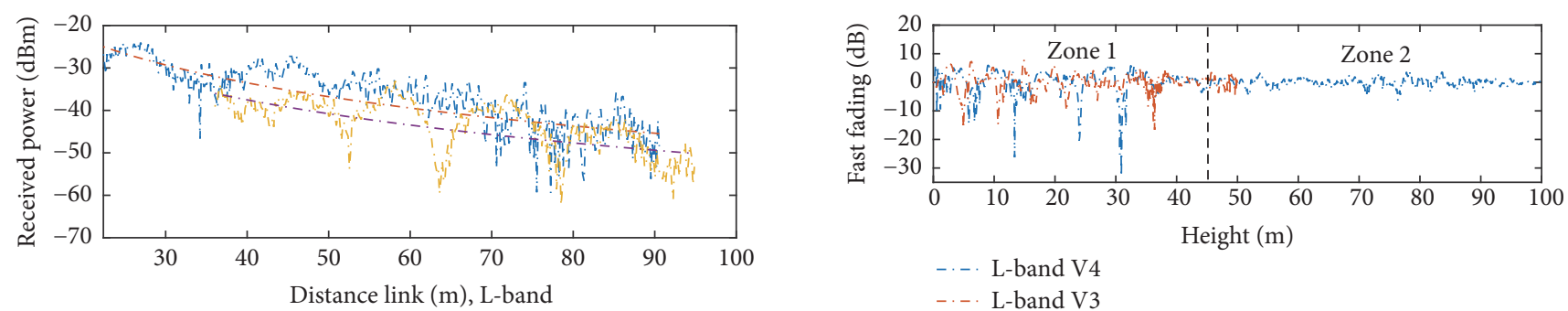

-... L-band V4

-... L-band V3
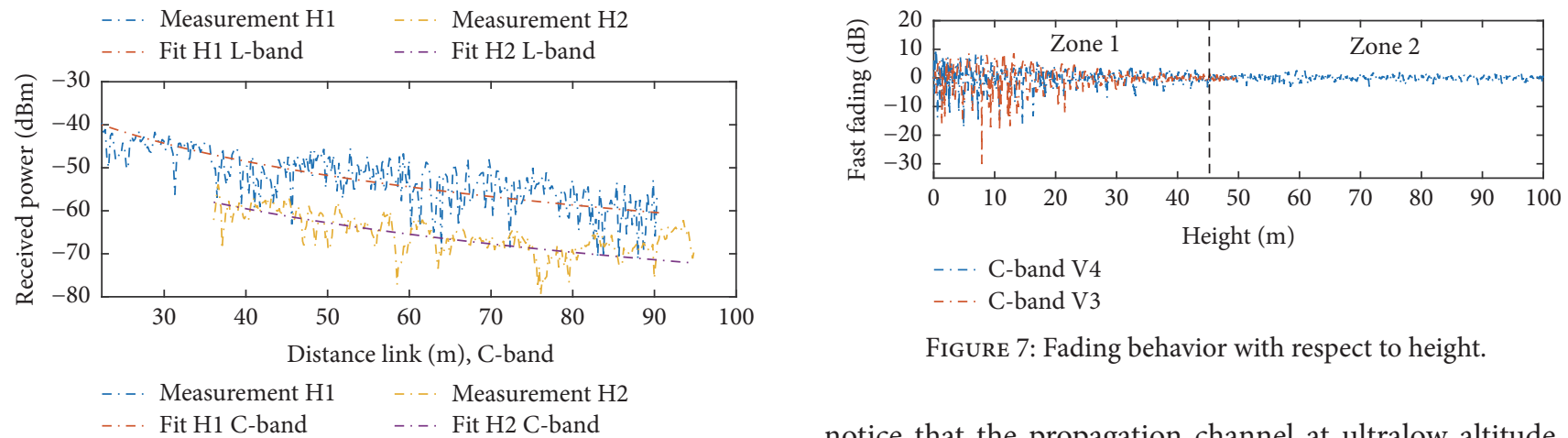

FIGURE 7: Fading behavior with respect to height.

FIGURE 5: Influence of the link distance on path loss for L-band/Cband.
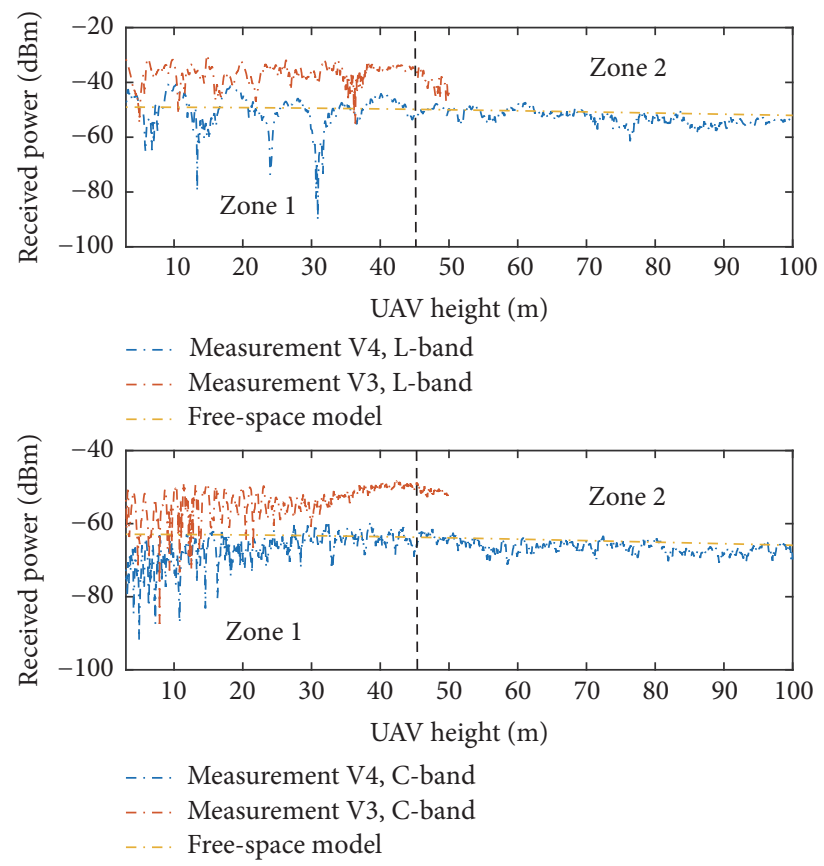

notice that the propagation channel at ultralow altitude is easily affected by those obstacles on the ground. The signal received is considered as a composition of a large number of waves originated from the LoS path, multipath, scattering, and even diffractions. This will challenge the communication for commercial UASs to carry out multiple missions in complex environments with strict regulations on flying distance and height.

In L-band/C-band, the fading depths and fading periods are different, which indicates that the decay of multipath is also related to the carrier frequency. The modeling of the small-scale fading is introduced in the following section using Rice statistics to model Rician $K$ factor with respect to height.

3.3. Small-Scale Fading. The Rician $K$ factor is an important parameter to describe the signal behavior, which has a significant influence on the channel capacity. It is a measure of the severity of fading where there is a LoS path from the Tx to the Rx. Generally, the Rician $K$ factor is modeled either as a temporal variation on the fixed wireless links [20] or as distance-dependent [21].

3.3.1. Estimation of Rician $K$ Factors. Rician distribution is normally used to describe the small-scale fading when there is a dominant component such as a LoS path. In our case, the UAV is in LoS during the whole measurements, and thus the Rician $K$ factor is suitable to evaluate the fading behavior. Figure 8 gives the probability density functions of measurements $\mathrm{V} 4$ and $\mathrm{H} 2$ for L-band/C-band using data normalized by a 20-wavelength root mean square [21], which are well fitted in Rician distributions. $K$ factor is estimated using the momentmethod approach [22].

$$
\begin{aligned}
K & =\frac{|\xi|^{2}}{\sigma^{2}} \\
|\xi|^{2} & =\left[G_{a}{ }^{2}-G_{v}{ }^{2}\right]^{1 / 2} \\
\sigma^{2} & =G_{a}-|\xi|^{2},
\end{aligned}
$$
lots of trees). According to our measurement results, we 

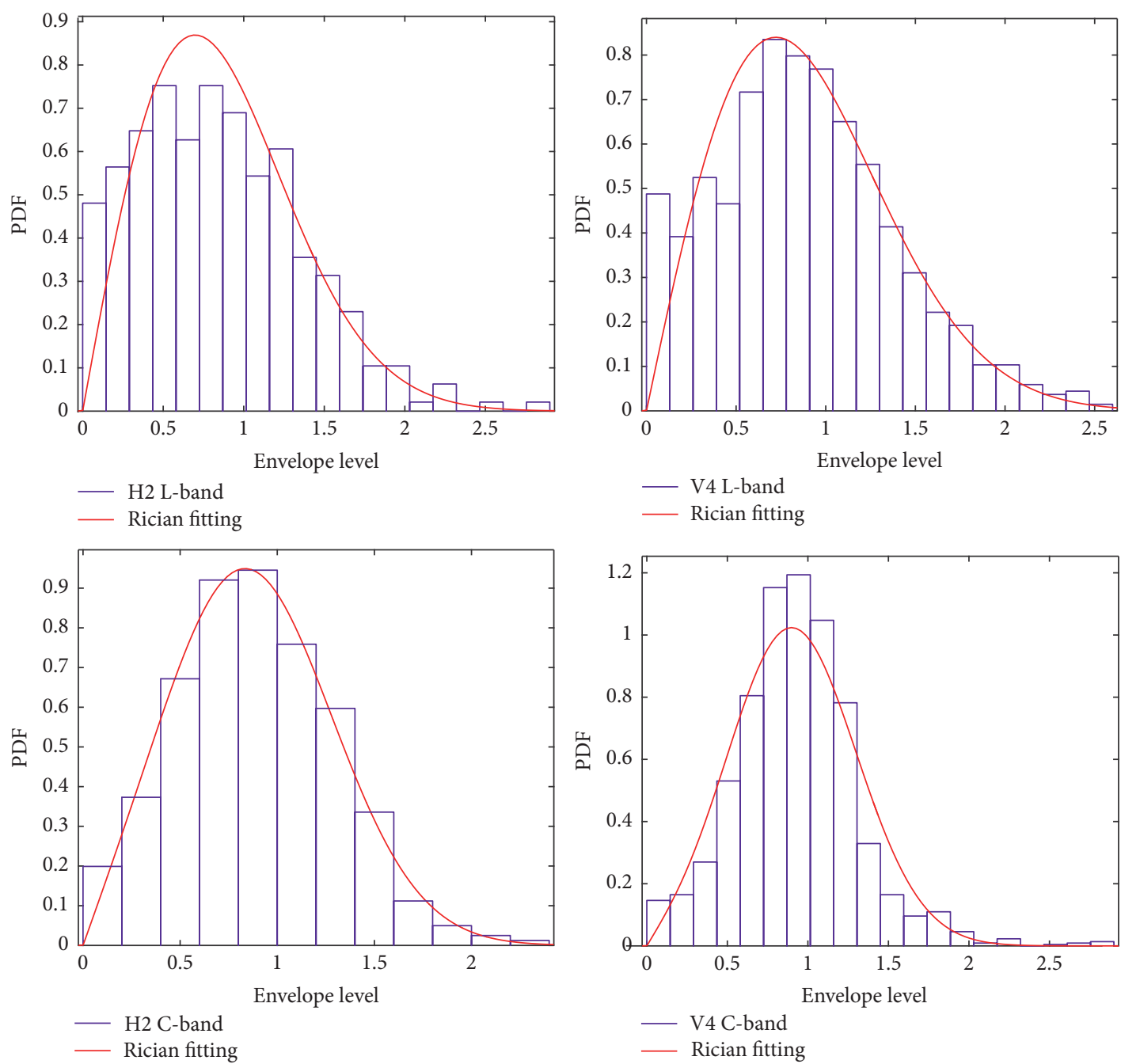

Figure 8: Probability density functions of the measurements.

where $G_{a}$ is the first moment of the power gain $(G)$ (which can be calculated by finite data) and $G_{v}$ is the second moment of $G$ about $G_{a}$ indicating the root mean square (RMS) fluctuation. The RMS is set at a length of 20 wavelengths. $K$ factor is calculated every $5 \mathrm{~m}$, which contains around 60 samples in average and is considered large enough to obtain correct results in our test environment [23].

3.3.2. Rician $K$ Factors Modeling. In [24], the author proposed a Rician $K$ factor model for a UAV channel based on measurement at altitudes from 500 to $2000 \mathrm{~m}$. The model considers the $K$ factor as a linear function of distance and the author states that the $K$ factor is nearly constant in his measurement.

$$
K(R)=K_{0}+n_{K}\left(R-R_{\min }\right)+Y,
$$

where $K_{0}$ is a constant value for distance $R_{\min }$ and $Y$ is the zero-mean Gaussian random variable with standard deviation $\sigma_{Y}$. We fit the parameters of this model based on the measurements at heights of $10 \mathrm{~m}(\mathrm{H} 1)$ and $30 \mathrm{~m}(\mathrm{H} 2)$, respectively, for L-band/C-band. The results are given in Table 3.
TABLE 3: Fitting parameters of model in (3) based on horizontal measurements $\left(R_{\min }=30 \mathrm{~m}\right)$.

\begin{tabular}{lcccccc}
\hline Band & Height & $n_{K}$ & $K_{0}$ & $\sigma_{Y}$ & Average & Estimation \\
\hline \multirow{2}{*}{ L-band } & $10 \mathrm{~m}$ & 0.0055 & 4.29 & 3.40 & 4.46 & 3.60 \\
& $30 \mathrm{~m}$ & 0.0082 & 7.28 & 4.13 & 7.50 & 7.25 \\
\hline \multirow{2}{*}{ C-band } & $10 \mathrm{~m}$ & 0.0805 & 3.52 & 2.88 & 5.80 & 3.03 \\
& $30 \mathrm{~m}$ & 0.0136 & 9.62 & 3.15 & 9.98 & 11.42 \\
\hline
\end{tabular}

The triangle dots in Figure 9 denote the statistic $K$ factors of measurements $\mathrm{H} 1$ and $\mathrm{H} 2$, and the straight lines are fittings of the model shown in (3). As observed, the $K$ factor is almost constant during the horizontal flights. However, the horizontal $K$ factor varies at different heights with a difference of $3-8 \mathrm{~dB}$.

According to the analysis of $K$ factor for different flying trajectories, it is convincing that the fading behavior is more related to the flying height instead of the distance at ultralow altitude. When the altitude of UAV is very low, the scatterers near the ground give strong multipath fading to the channel. 

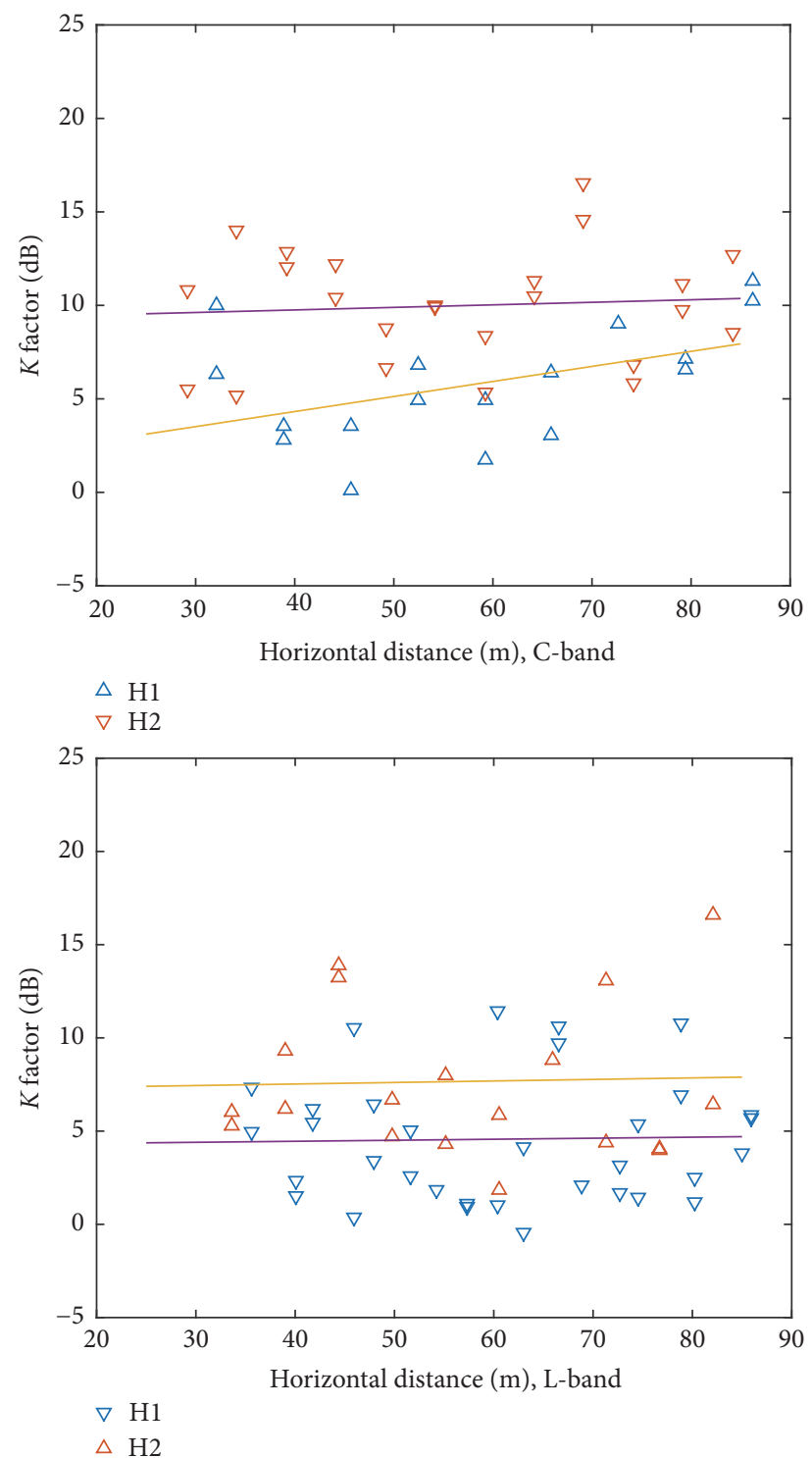

FIgURE 9: Rician $K$ factor with respect to the horizontal distance for L-band/C-band.

When the UAV gets higher, the effects of scatterers mitigate. Once the UAV reaches above BP, the channel gets rid of the reflection from scatterers on the ground, and hence the quality of the channel improves greatly and approaches the free-space model. Considering that the fading characteristics rely on height instead of the elevation angle or the distance, a height-dependent Rician $K$ factor model is proposed as follows:

$$
K(R)= \begin{cases}C_{1}+n_{1} h+Y_{1} & h<\mathrm{BP}, \\ C_{2}+n_{2} h+Y_{2} & h>\mathrm{BP} .\end{cases}
$$

The parameters fitted are given in Table 4 .

$K$ factor in the last column of Table 3 is estimated using the proposed model in (4). Comparing it to the average $K$ factor calculated by horizontal measurements at $10 \mathrm{~m}$ and
TABLE 4: Parameters of the proposed $K$ model at L-band/C-band.

\begin{tabular}{lcccccc}
\hline \multirow{2}{*}{ Band } & \multicolumn{3}{c}{ Height < BP } & \multicolumn{3}{c}{ Height $>\mathrm{BP}$} \\
& $C_{1}$ & $n_{1}$ & $\sigma_{Y_{1}}$ & $C_{2}$ & $n_{2}$ & $\sigma_{Y_{2}}$ \\
\hline L-band & 1.77 & 0.1829 & 2.65 & 9.52 & 0.0294 & 2.14 \\
C-band & -1.17 & 0.4195 & 3.68 & 14.38 & 0.0345 & 2.93 \\
\hline
\end{tabular}

$30 \mathrm{~m}$ heights, we can observe that the estimation is good enough and the error is very small.

The distributions of $K$ factor are depicted in Figure 10, which clearly shows high dependency on height. It also shows two tendencies in two zones, and the BP estimated is around $50 \mathrm{~m}$ for L-band and $40 \mathrm{~m}$ for C-band based on the least square method. This is due to the lower propagation losses at lower frequencies, which allows higher order reflections to affect propagation.

The cumulative probability distributions of the $K$ factors of all measurements are illustrated in Figure 11, which clearly reveals the effect of height and the difference of the two frequency bands. Generally, the $K$ factor in the $\mathrm{C}$-band is higher than that in the L-band and is increasing before BP, while after BP it tends to be constant.

\section{Validation of Measurements by Simulation}

In this section a simulation was performed to explain the underlying propagation mechanism of the "breakpoint," yet more measurements under different scenarios are still necessary for further verification.

Assuming there exists a reflection from the sidewall of the obstacle as depicted in Figure 12, we get a "reversed" two-ray model, where $H_{b}$ is the height of the obstacle and $h_{r}$ is the height of the reflection point. During the ascent process of UAV, the reflection point is synchronously moving up until the UAV reaches a certain height, after which the reflection point disappears. Such behavior can result in a decrease of multipath components, which meets our observations in measurements. Assuming the distance from the Tx and the $\mathrm{Rx}$ to the reflecting plane of the obstacle is $d_{t}$ and $d_{r}$, respectively, by ignoring the height of $\mathrm{Rx}$, which is much lower than the obstacle, $h_{r}$ can be calculated as

$$
h_{r}=\frac{d_{r} \cdot h}{d_{r}+d_{t}} .
$$

Modified from the flat earth two-ray (FE2R) model given in [25], the multiray model for $N$ paths is calculated as

$$
P_{r}=P_{t}\left(\frac{\lambda}{4 \pi}\right)\left|\frac{\sqrt{G_{\mathrm{LoS}}}}{l_{\mathrm{LoS}}}+\sum_{i=1}^{N} \frac{R_{i} \sqrt{G_{\mathrm{NLoS} i}} e^{-j \Delta \phi_{i}}}{l_{\mathrm{NLoS} i}}\right|^{2}
$$

where $P_{t}$ is the transmitting power, $G_{\mathrm{LoS}}$ and $G_{\mathrm{NLoS}}$ are the total antenna gain for LoS ray and for NLoS ray, respectively, $l_{\mathrm{LoS}}$ is the traveling distance of the LoS path, and $l_{\mathrm{NLOS}}$ is the traveling distance of NLoS paths. 

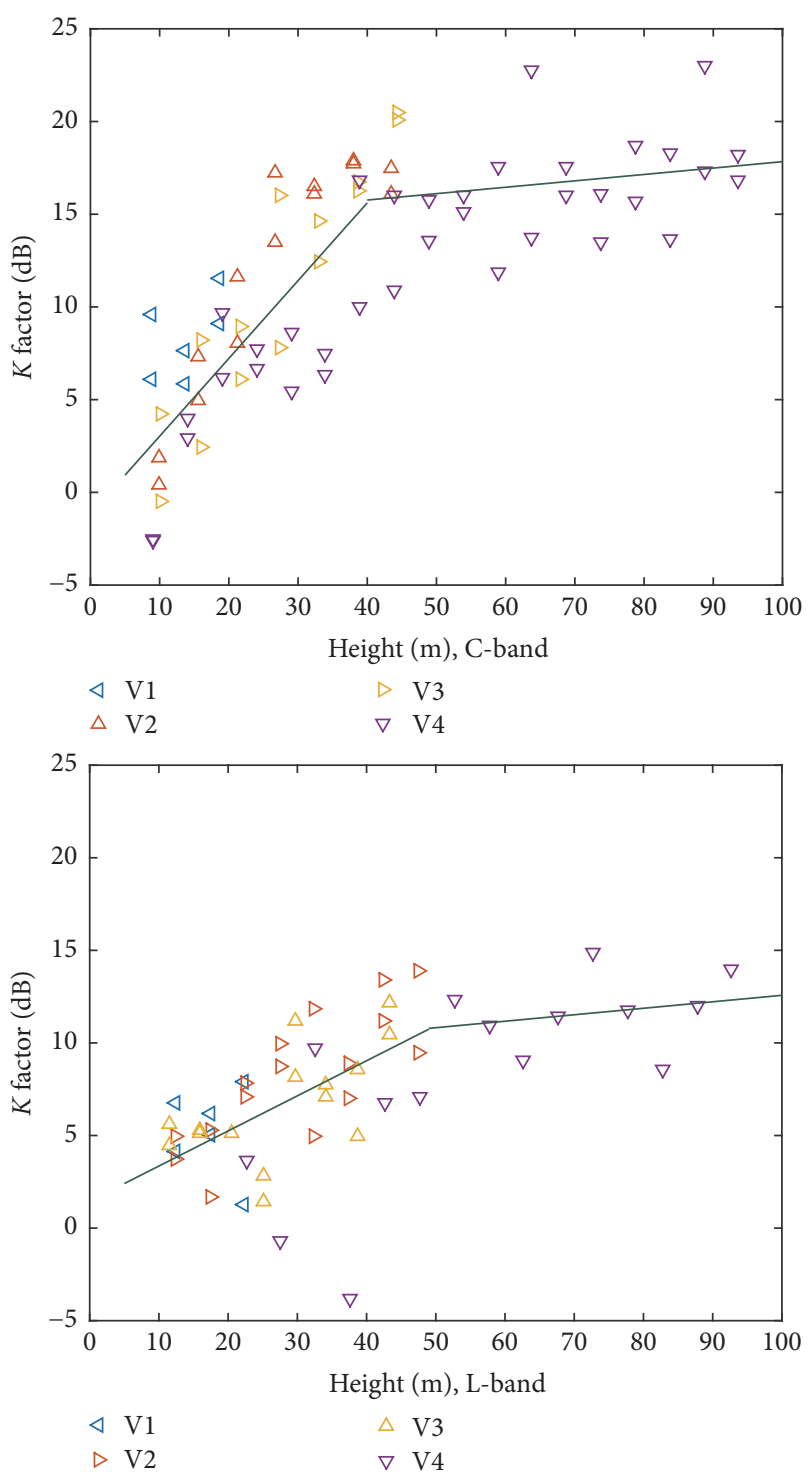

FIGURE 10: Rician $K$ factor with respect to the heights for L-band/Cband. Lines refer to the piecewise linear fitting of $K$ factor.

In (6), $R_{i}$ is the reflecting coefficient for $i$ path which depends on the impinging angle and dielectric constant $\varepsilon_{r}$.

$$
R=\frac{\sin \phi-Z}{\sin \phi+Z},
$$

where $Z$ is written as

$$
Z= \begin{cases}\frac{\sqrt{\varepsilon_{r}-\cos ^{2} \theta}}{\varepsilon_{r}} & \text { for vertical polarization, } \\ \sqrt{\varepsilon_{r}-\cos ^{2} \theta} & \text { for horizontal polarization. }\end{cases}
$$

The simulation results were computed based on the FE2R model and multiray model given in (6).

Considering the fact that there are many obstacles in our measurement environment, the power we received can

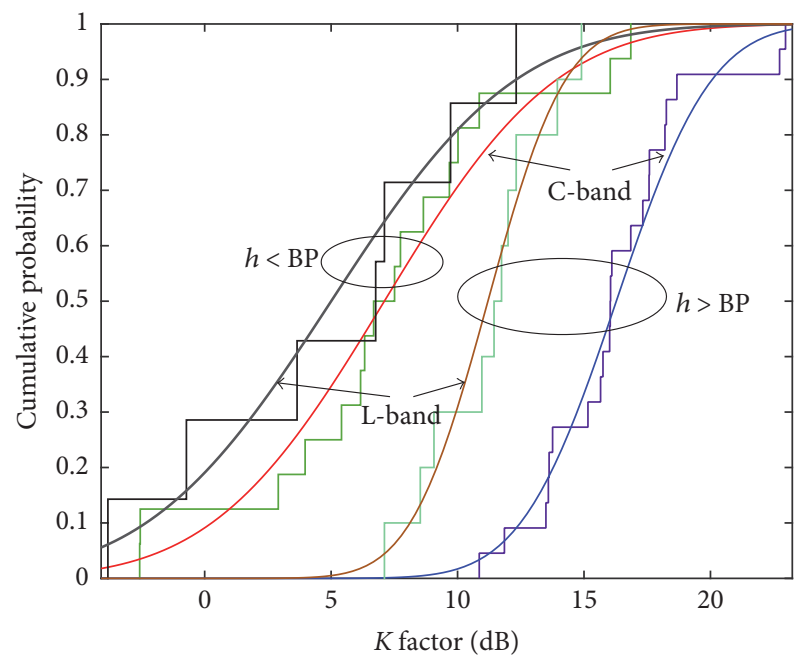

FIGURE 11: The distribution of Rician $K$ factor for L-band/C-band.

be regarded as a composition of multiple propagation rays, including the LoS component, the components caused by ground reflection, and the reflections from obstacles as well as the scattering and diffractions. Thus, four major obstacles considered to contribute the most to the power received as illustrated in Figure 13 are included in the simulation.

The major problem for the simulation is that it is difficult to find the exact reflecting point of different objects, especially for the containers since there were many containers and they were moving during the days when the measurements were conducted. It is noticed that, in the simulation, the distance between the reflecting surface and the $\mathrm{Tx} / \mathrm{Rx}$ as well as the incident angle has a significant impact on the decaying behavior of received power as depicted in the multiray model, while the reflection coefficient determines the fading depth of the interference.

The dielectric constants of the buildings are set according to $[26,27]$. The coefficient $\varepsilon_{r}$ of the cement concrete, which is the main material of buildings, can vary from 4 to 28 . Considering the aging process of buildings and the influence of frequency on the dielectric constant, $\varepsilon_{r}$ of building 1, building 2, and building 3 are all set to 7. Normally, the dielectric constant $\varepsilon_{r}$ of the grassland depends on the actual water content and the thickness of the grass. In our environment, the ground is a dry grassland which cannot serve as an ideal reflection surface; therefore, the value of $\varepsilon_{r}$ for the ground reflection is set to 5 in our case.

As observed from the graph in Figure 6, the maximum fading depth reaches $40 \mathrm{~dB}$, which is the result of a very high reflection coefficient. Our study shows that only if the dielectric constant $\varepsilon_{r}$ is set to more than 10000 can the fading depth reach $40 \mathrm{~dB}$. However, the ground and the buildings in the environment cannot provide such a high reflection coefficient, and thus it is postulated that such a phenomenon was caused by the reflection from the metal containers nearby. Since the dielectric constant of the ideal conductor is close to infinity, the coefficient $\varepsilon_{r}$ of the containers which are made of steel can be very high. 


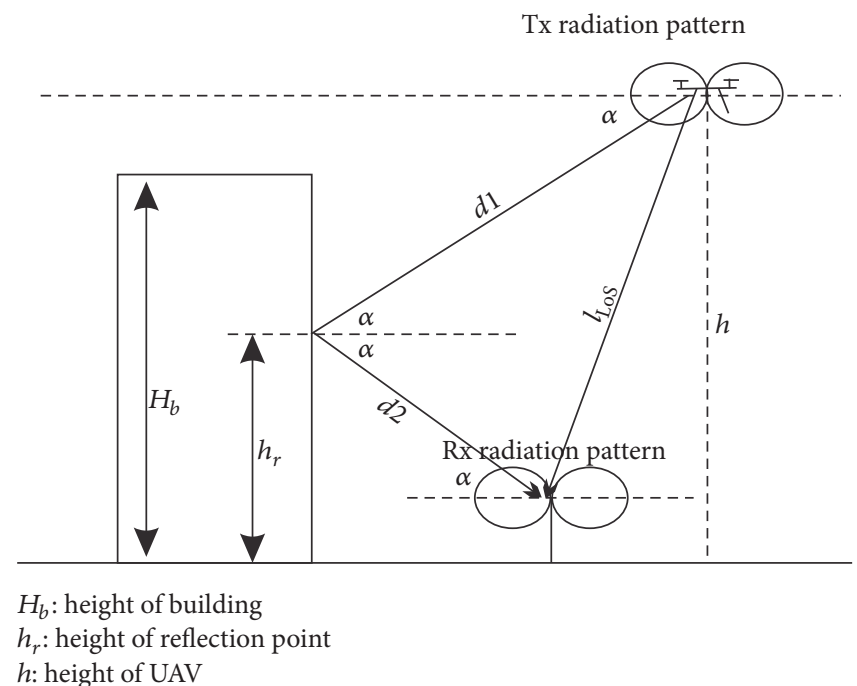

FIGURE 12: Sketch of the reflection from the sidewall.

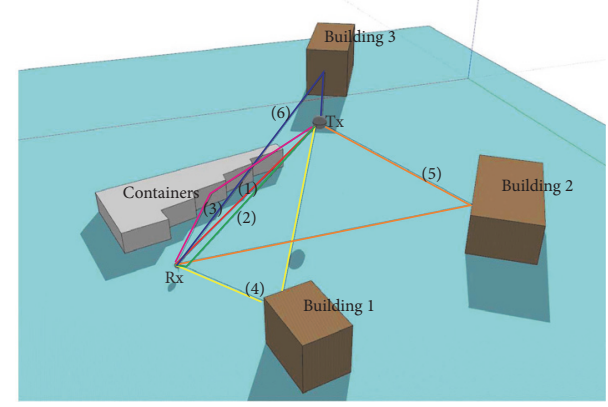

(1) LoS

(2) Ground reflection

(3) Reflection from containers

(4) Reflection from Building 1

(5) Reflection from Building 2

(6) Reflection from Building 3

FIGURE 13: Sketch of the simulated environment.

In the simulation, we only take into account the LoS and the single-bounce reflections. The simulated environment is in accordance with the test environment shown in Figure 2. The heights are given in Table 6, and the dielectric constants are listed in Table 5. The antennas used in the simulation are omnidirectional and vertically polarized in the elevation plane.

Assuming that the reflections from the obstacles gradually decrease when the UAV ascends, then the disappearing heights for reflections from containers and building 1 , building 2, and building 3 are $30 \mathrm{~m}, 40 \mathrm{~m}, 50 \mathrm{~m}$, and $60 \mathrm{~m}$, respectively.

The simulation results for L-band/C-band are depicted in Figure 14. The red dash-dotted line represents the received power generated from typical flat earth 2-ray (FE2R) model, while the blue solid line gives the result of superposition of the LoS path, the ground reflection, and the singlebounce reflections from containers, building 1, building 2 ,

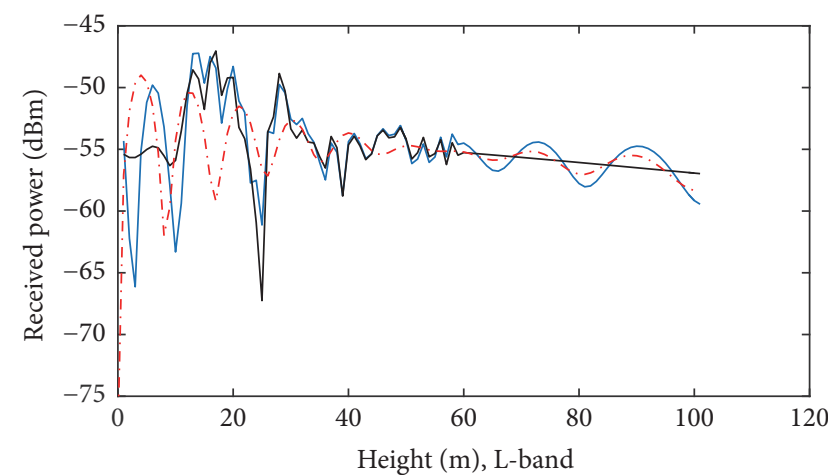

- Case 1: with ground reflection

— Case 2: without ground reflection

-... Case 3: FE2R model

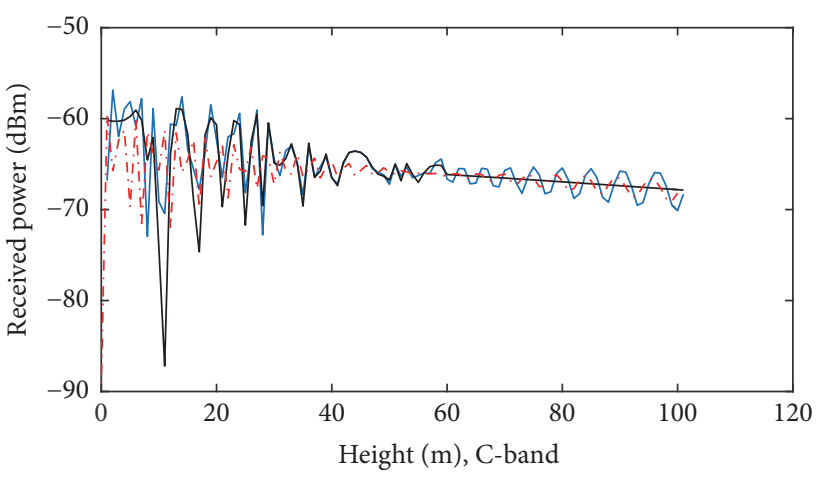

- Case 1: with ground reflection

- Case 2: without ground reflection

-. - Case 3: FE2R model

FIGURE 14: Simulation results for L-band/C-band.

and building 3, respectively. Note that the black curve is generated under the condition basically the same as case 3, only without the ground reflection.

We can observe from Figure 14 a similar pattern of power variation to the empirical results depicted in Figure 6. In the 
TABLE 5: The configurations of the simulation.

Coefficient $\varepsilon_{r}$

\begin{tabular}{lccc} 
Ground (dry grass) & Containers (steel) & $\begin{array}{c}\text { Buildings 1, 2, and } \\
3 \\
\text { (cement bricks) }\end{array}$ & Antennas \\
\hline 5 & 10000 & 7 & $\begin{array}{c}\text { Omnidirectional, vertically polarized in } \\
\text { elevation }\end{array}$ \\
\hline
\end{tabular}

TABLE 6: The relative height of the objects neighborhood.

\begin{tabular}{lccc}
\hline Building 1 & Building 2 & Building 3 & Containers \\
\hline $14 \mathrm{~m}$ & $13 \mathrm{~m}$ & $30 \mathrm{~m}$ & $9 \mathrm{~m}$ \\
\hline
\end{tabular}

simulation, the received power at first consists of the LoS and the reflections from containers, building 1, building 2, and building 3. When the UAV is above the disappearing altitude of container reflection, the received power contains only the LoS and the reflections from buildings. With further increasing of UAV height, less and less reflection remains until finally the LoS is the only path existing.

This simulation only considers an ideal simplified scenario with objects with smooth surfaces. Multiple-bounce reflections as well as diffractions are not taken into consideration and therefore there are some inconsistencies between the simulation results and measurements. However, the overall tendencies of our simulation results show a high similarity to the empirical measurement results: it can be observed that two propagation zones with different fading characteristics exist at both simulations and measurements. Nevertheless, more measurements are necessary to investigate the details of the mechanism leading to the "breakpoint" effect observed here.

\section{Conclusions}

In this paper, a measurement campaign dedicated to characterizing AG channels has been introduced. We have noticed that the complex scatterers near the ground play an important role in the characterization of the propagation channel. Multiple measurements on different positions were conducted, and the analysis of the measurements indicates that the channel shows an obvious dependency on the height instead of the elevation angle or the distance. Path loss has been modeled and small-scale fading has been investigated. The path loss exponents reveal the channel's dependency on height which is previously seldom investigated. The fading behavior is distinguished obviously in different ranges of heights, and thus a height-dependent Rician $K$ factor model is proposed, which will be useful in the modeling of UAV channels and the performance analysis of the UAS wireless communication systems such as the channel capacity and the throughput.

An interesting change of the propagation channel after the so-called "breakpoint" was observed, and a geometrybased simulation was performed. However, more measurements under different scenarios are necessary to accurately investigate the underlying mechanism of the "breakpoint," which is considered to be useful for guiding the UAVs to find the best communication altitude to avoid deep fading when flying over a given environment.

\section{Disclosure}

This work was done when Z. Qiu and X. Chu were visiting UPM as part of the Double Master Degree Program between Tongji University and the Technical University of Madrid, supported by the China Scholarship Council (CSC).

\section{Conflicts of Interest}

The authors declare that there are no conflicts of interest regarding the publication of this paper.

\section{Acknowledgments}

This work was developed under the framework of ENABLING 5G TEC2014-55735-C3-2-R funded by the Spanish Ministry of Economy and Competitiveness and was jointly supported by the Key Project " $5 \mathrm{G} \mathrm{Ka}$ frequency bands and higher and lower frequency band cooperative trail system research and development" under Grant 2016ZX03001015 of China Ministry of Industry and Information Technology and the National Natural Science Foundation of China general project under Grant 61471268.

\section{References}

[1] M. Bedford and A. Hanscom, "Unmanned aircraft system (UAS) service demand 2015-2035, literature review projections of future usage," Tech. Rep., US Department of Transportation, Technical, 2013.

[2] Federal Aviation Administration (FAA), "FAA Modernization and Reform Act of 2012 (FMRA)," pub. L. No. 112-95 331, 126 Stat. 11,72 (2012), 334(c) (2) (C).

[3] European Aviation Safety Agency, "Civil drones (Unmanned aircrafts)," https://www.easa.europa.eu/easa-and-you/civil-dronesrpas.

[4] "Characteristics of unmanned aircraft systems and spectrum requirements to support their safe operation in non-segregated airspace, ITU-R," ITU-R, Report ITU-R M.2171, 2009, https:// www.itu.int/en/ITU-R/space/snl/Documents/R-REP-M.21712009-PDF-E.pdf.

[5] J. D. Parsons, The Mobile Radio Propagation Channel, John Wiley \& Sons, New York, NY, USA, 2000.

[6] D. W. Matolak and R. Sun, "Air-ground channel measurements \& modeling for UAS," in Proceedings of the 2013 13th Annual 
Integrated Communications, Navigation and Surveillance Conference, ICNS 2013, USA, April 2013.

[7] F. Ono, K. Takizawa, H. Tsuji, and R. Miura, "S-band radio propagation characteristics in urban environment for unmanned aircraft systems," in Proceedings of the International Symposium on Antennas and Propagation, ISAP 2015, November 2015.

[8] M. Simunek, F. P. Fontán, and P. Pechac, "The UAV low elevation propagation channel in urban areas: Statistical analysis and time-series generator," IEEE Transactions on Antennas and Propagation, vol. 61, no. 7, pp. 3850-3858, 2013.

[9] T. J. Willink, C. C. Squires, G. W. Colman, and M. T. Muccio, "Measurement and characterization of low-altitude airto-ground MIMO channels," IEEE Transactions on Vehicular Technology, vol. 65, no. 4, pp. 2637-2648, 2016.

[10] A. Kezuka, S. Saito, T. Yoshihara, and S. Saitoh, "Effect of building on VHF propagation above airport surface," in Proceedings of the 21st International Symposium on Antennas and Propagation, ISAP 2016, pp. 216-217, October 2016.

[11] A. Al-Hourani, S. Kandeepan, and A. Jamalipour, "Modeling air-to-ground path loss for low altitude platforms in urban environments," in Proceedings of the 2014 IEEE Global Communications Conference, GLOBECOM 2014, pp. 2898-2904, December 2014.

[12] E. Yanmaz, R. Kuschnig, and C. Bettstetter, "Channel measurements over 802.11a-based UAV-to-ground links," in Proceedings of the 2011 IEEE GLOBECOM Workshops, pp. 1280-1284, December 2011.

[13] N. Goddemeier, K. Daniel, and C. Wietfeld, "Role-based connectivity management with realistic air-to-ground channels for cooperative UAVs," IEEE Journal on Selected Areas in Communications, vol. 30, no. 5, pp. 951-963, 2012.

[14] W. Newhall, R. Mostafa, C. Dietrich et al., "Wideband air-toground radio channel measurements using an antenna array at $2 \mathrm{GHz}$ for low-alititude operations," in Proceedings of the IEEE Military Communications Conference, 2003. MILCOM 2003., pp. 1422-1427, Boston, MA, USA.

[15] Q. Feng, J. McGeehan, E. K. Tameh, and A. R. Nix, "Path loss models for air-to-ground radio channels in urban environments," in Proceedings of the 2006 IEEE 63rd Vehicular Technology Conference, VTC 2006-Spring, pp. 2901-2905, Australia, July 2006.

[16] W. Khawaja, I. Guvenc, and D. Matolak, "UWB channel sounding and modeling for UAV air-to-ground propagation channels," in Proceedings of the 59th IEEE Global Communications Conference, GLOBECOM 2016, USA, December 2016.

[17] X. Cai, A. Gonzalez-Plaza, D. Alonso et al., "Low altitude UAV propagation channel modelling," in Proceedings of the 2017 11th European Conference on Antennas and Propagation (EuCAP), pp. 1443-1447, Paris, France, March 2017.

[18] R. He, Z. Zhong, B. Ai, and J. Ding, "An empirical path loss model and fading analysis for high-speed railway viaduct scenarios," IEEE Antennas and Wireless Propagation Letters, vol. 10, pp. 808-812, 2011.

[19] D. W. Matolak and R. Sun, "Air-Ground Channel Characterization for Unmanned Aircraft Systems-Part III: The Suburban and Near-Urban Environments," IEEE Transactions on Vehicular Technology, vol. 66, no. 8, pp. 6607-6618, 2017.

[20] L. J. Greenstein, S. S. Ghassemzadeh, V. Erceg, and D. G. Michelson, "Ricean $\mathrm{K}$-factors in narrow-band fixed wireless channels: theory, experiments, and statistical models," IEEE Transactions on Vehicular Technology, vol. 58, no. 8, pp. 40004012, 2009.
[21] R. He, Z. Zhong, B. Ai, and J. Ding, "Distance-dependent model of ricean k-factors in high-speed rail viaduct channel," in Proceedings of the 76th IEEE Vehicular Technology Conference, VTC Fall 2012, Canada, September 2012.

[22] L. J. Greenstein, D. G. Michelson, and V. Erceg, "Momentmethod estimation of the Ricean $K$-factor," IEEE Communications Letters, vol. 3, no. 6, pp. 175-176, 1999.

[23] W. C. Y. Lee, "Estimate of local average power of a mobile radio signal," IEEE Transactions on Vehicular Technology, vol. 34, no. 1, pp. 22-27, 1985.

[24] D. W. Matolak and R. Sun, "Air-Ground Channel Characterization for Unmanned Aircraft Systems-Part I: Methods, Measurements, and Models for Over-Water Settings," IEEE Transactions on Vehicular Technology, vol. 66, no. 1, pp. 26-44, 2017.

[25] A. Goldsmith, Wireless Communications, Cambridge University Press, New York, NY, USA, 1st edition, 2005.

[26] A. Martinez, A. P. Byrnes et al., Modeling dielectric-constant values of geologic materials: an aid to ground-penetrating radar data collection and interpretation, Kansas Geological Survey Lawrence, Kansas, Kan, USA, 2001.

[27] L. Shi, Q. Xu, B. Chen, and C. Gao, "Measurement of the frequency-dependent dielectric constant of concrete materials by TDR and wavelet modeling method," in Proceedings of the 3rd Asia-Pacific Conference on Environmental Electromagnetics, CEEM 2003, pp. 626-629, November 2003. 


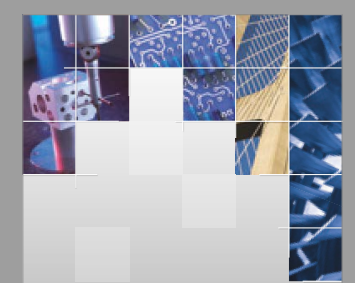

\section{Enfincering}
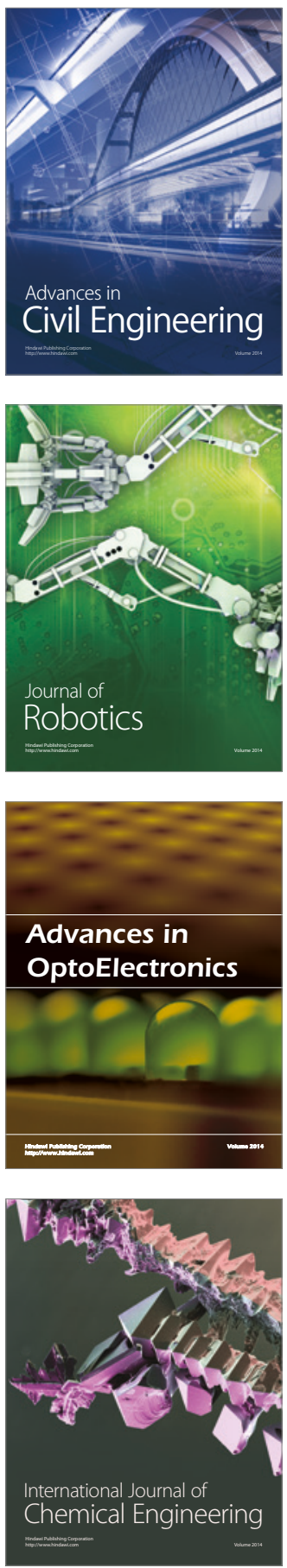

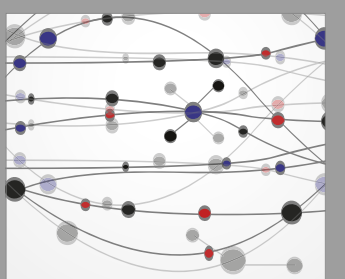

The Scientific World Journal

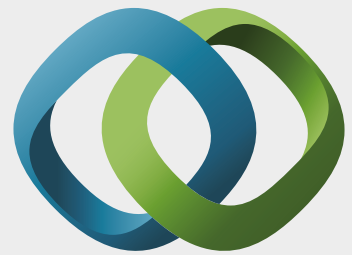

\section{Hindawi}

Submit your manuscripts at

https://www.hindawi.com
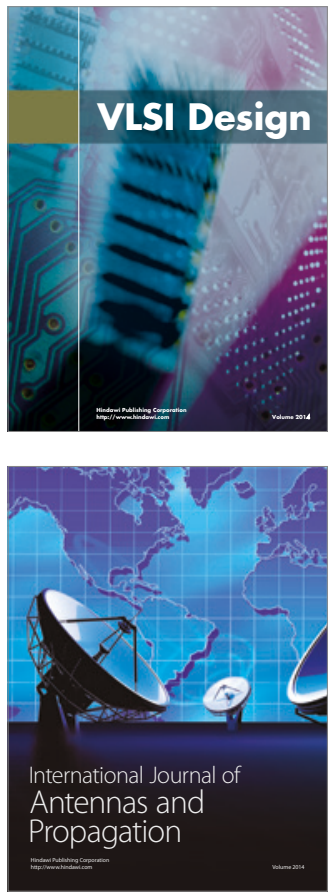

\section{Rotating}

Machinery
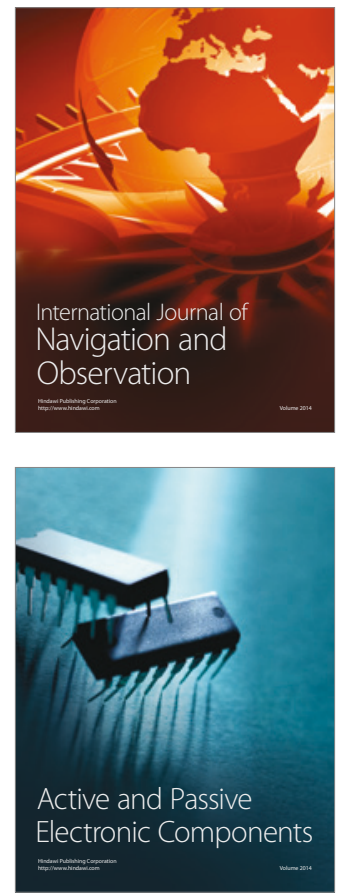
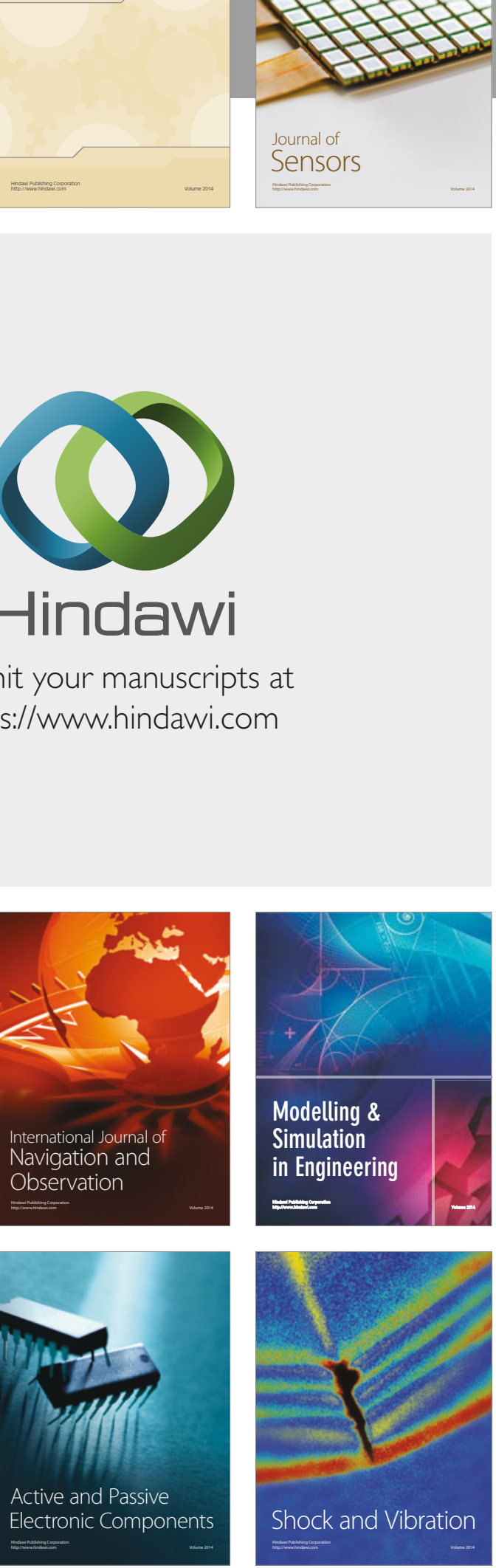
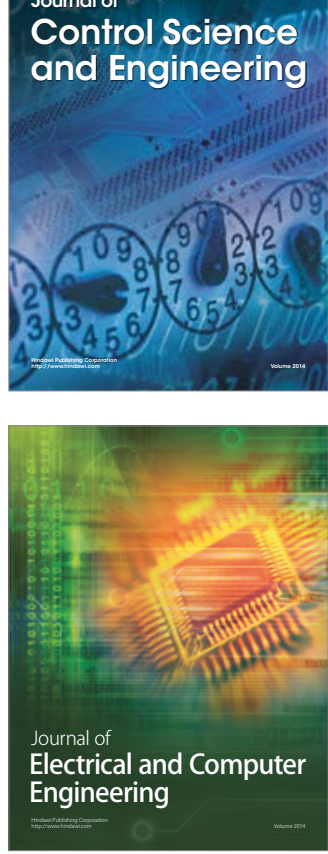

Distributed

Journal of

Control Science

and Engineering
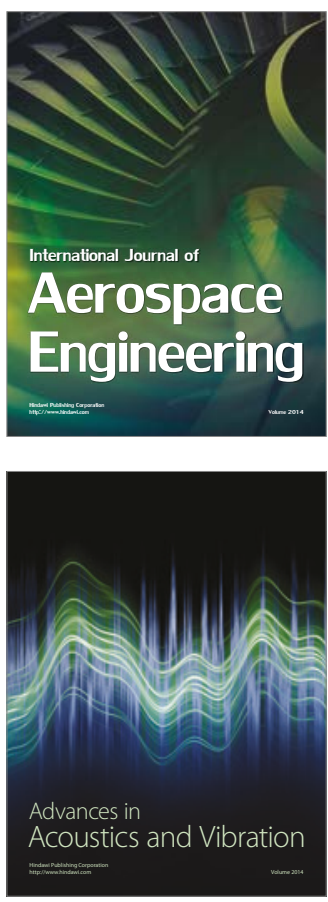

Sensor Networks 